\title{
Newborn hearing screening project using transient evoked otoacoustic emissions: Western Sicily experience
}

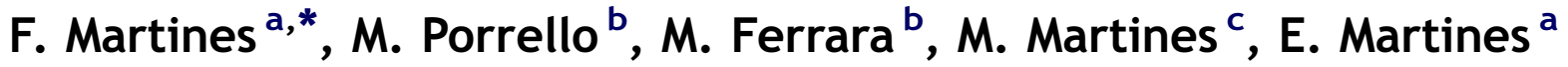 \\ ${ }^{a}$ Università degli Studi di Palermo, Dipartimento di Biotecnologie Mediche e Medicina Legale, \\ Sezione di Audiologia, Italy \\ ${ }^{\mathrm{b}}$ Azienda Ospedaliera "O.C.R." di Sciacca, U.O. di Pediatria/Neonatologia, Italy \\ ' Università degli Studi di Palermo, Dipartimento Materno Infantile, Sezione di Pediatria, Italy
}

Received 24 January 2006; received in revised form 27 September 2006; accepted 30 September 2006

\section{KEYWORDS \\ Neonatal screening; Sensorineural hearing loss; \\ Otoacoustic emissions}

\begin{abstract}
Summary
Objective: To study the incidence of congenital sensorineural hearing loss in all newborns introducing a screen test with a protocol no expensive, with a good "screen sensitivity" that could let an earlier identification of hearing impairment beginning early intervention by 2 months of age and increasing the probability of having language development within the normal range of development.

Methods: The study was conducted in Sciacca hospital from the beginning of 2003 to our days and was carried out with transient evoked otoacoustic emission using the criteria for PASS or RETEST and considering eventual prenatal and perinatal risk factors. All the newborns were divided into four groups each one with its personal secondary step program.

Results: In the years 2003-2004 the number of the newborns in Sciacca hospital was: 538 for 2003, 653 for 2004 with a total of 1191; all these infants were divided in three groups: resident in Sciacca, resident in the Sciacca borderlands and resident out of the district of Sciacca. The coverage (percentage of the target population who undergo the screen) was of 90\% in the 2003 (483 newborns) and of 90\% in the 2004 (585 newborns) with two cases of congenital sensorineural hearing loss identified.

The incidence of sensorineural hearing loss, in the District of Sciacca, was estimated to be 2.07/1000 in 2003 and 1.70/1000 in 2004.

Conclusions: The higher incidence of sensorineural hearing loss in our study is due to a high prevalence of consanguineous marriage in Sicily that was shown to be linked with hearing impairment.
\end{abstract}

\footnotetext{
* Corresponding author. Tel.: +39091 545666; fax: +39 0916554271.

E-mail address: francescomartines@hotmail.com (F. Martines).
} 
The "sensitivity value" was 95\% at the first step but became $99 \%$ after the second step with a few number of false positive $(0.74 \%)$.

All the infants with a diagnosis of sensorineural hearing loss began a rehabilitation program before the age of 5 months and they have a good speech development and speech intelligibility.

(C) 2006 Elsevier Ireland Ltd. All rights reserved.

\section{Introduction}

Hearing loss is one of the commonest disorders present at birth; the literature report a large range of childhood hearing impairment (PCHI) from 0.7 to $4.2 / 1000$ live births [1-16]; it is probably due to many elements that each author studied:

- unilateral or bilateral hearing loss;

- severity of hearing loss;

- age, sex, ethnicity and socio-economic status in a particular population;

- presence in the group observed of prenatal and perinatal risk factors.

However, it should be mentioned that if we considered all the newborns to estimate the PCHI incidence, this value should be underestimated.

It has long been believed that an earlier identification of hearing impairment must lead to better outcomes, and currently there is a reliable evidence that this is the case in the domains of communication, educational achievement and quality of life [17-20].

The reason of this way of thinking is founded on the fact that although the development of the cochlea is almost complete at birth the inner ear active mechanisms need a further maturation, a process which is related to and dependent upon auditory stimulation [21]. Thus a congenital permanent hearing impairment (PHI) is likely to result in physiological deprivation unless compensated and may result in insufficient development of the central auditory structures [22,23].

Knowledge on the prevalence of congenital permanent childhood hearing impairment, as well as later acquired $\mathrm{HI}$, forms the basis for the planning and provision of paediatric hearing health services and it is a strict requirement for the introduction of hearing screening as a public health matter.

Usually screening can be regarded as a specific medical intervention with the aim of detecting disease or a certain health condition in individuals who are not primarily suspected of having them [24]. In contrast to the usual diagnostic interventions, which are carried out when a condition is suspected or known to exist, screening is applied to a population regarded primarily as healthy; in our case the neonatal hearing screening procedure must be able to detect with adequate certainty the congenital hearing loss;

The American National Institutes of Health Consensus Statement [25] and more recently the European Consensus Statement [26] recommended the implementation of universal screening before 3 months of life.

Technological developments have led to the worldwide introduction of universal neonatal screens for hearing impairment so that the three techniques of evoked otoacoustic emissions $\left(E O A E_{s}\right)$, automated auditory brainstem response (AABR) and a combination [27-30], have now been accepted by Universal Newborn Hearing Screening (UNHS). All this techniques, that had many problems in the beginning, were developed by training and modifications of their own protocols.

Advantages of screening by $\mathrm{TEOAE}_{\mathrm{S}}$ include [31]:

1. a sophisticated examiner is not required to administer the test,

2. actual testing time per infant ranges from 1 to $5 \mathrm{~min}$,

3. acoustic stimuli is non-invasive and costs less than use of disposable electrodes and ear cushions.

Screening tests do not identify deafness but underline those individuals who demonstrate a greater probability of having a hearing loss so they may be further evaluated to determine if a hearing impairment does exist, and if so, its extent. Furthermore, false-positive and false-negative results are inevitable in any economically feasible procedure.

The aim of this work is to develop a neonatal screening, based on transient evoked otoacoustic emissions ( $\left(T_{E O A E_{S}}\right)$, introducing a screen test with a low-cost protocol and need no medical control, increasing options for successful treatment.

\section{Material and methods}

To reduce the number of the fugitives and to have an adequate coverage (percentage of the target population who undergo the screen), we though to begin this screening program in a province 
Table 1 Groups identified

\begin{tabular}{|c|c|}
\hline Group selected & Guideline \\
\hline \multicolumn{2}{|l|}{ PASS without risk factor } \\
\hline PASS with risk factor ${ }^{a}$ & Retest at the age of 7 months \\
\hline FAIL without risk factor & Re-screening after 2 weeks for a maximum of four times ${ }^{b}$ \\
\hline FAIL with risk factor & Retest after 2 weeks $^{b}$ \\
\hline
\end{tabular}

(Sciacca) in which there is only a paediatric unit; the families of the babies who were born in others hospitals were informed by paediatrics about this study and decided to undergo or not undergo $\mathrm{OAE}_{\mathrm{S}}$ screening.

The study was conducted in Sciacca hospital from the beginning of 2003 to our days and it was carried out with transient evoked otoacoustic emission using the PASS-RETEST criteria. Before the test was started, parents were informed about the purpose of the test and the possible outcome; this was done by providing written information.

To identified risk factors the mothers were interviewed about the presence of: family history of permanent childhood hearing impairment, intrauterine infection, ototoxic drugs administration, consanguinity. We also considered a perinatal risk factors: cranio-facial abnormality, birth weight $<1500$ g, hyperbilirubinaemia, low apgar scores, mechanical ventilation $\geq 5$ days and neonatal intensive care $>48 \mathrm{~h}[8,31-33]$. In this way we were able to divide all the newborns into two groups: newborn with risk factor and newborn without risk factor. The screening test was done 3 weeks after the birth (XX day), through an appointment given at the newborn dismission. We prefer to do the exam in the third week to avoid the false positive we could obtain in the first week of life, due to moisture, that is the birth debris and retained fluid in the ear canal.

The ECHOCHECK, that is based on the ILO88 system of TEOAE recording, was used for all testing. ILO ECP probe was used; the click rate was approximately $97 \mathrm{~s}^{-1}$ and each stimulus consisted of a single $80 \mu$ s square pulse. Stimuli are presented in blocks of four stimuli: three small positive polarity stimuli followed by one big negative polarity stimulus three times as large.

The response from the ear canal was then windowed and filtered to remove unwanted signals; all response data outside a window from $4 \mathrm{mS}$ to $10 \mathrm{mS}$, after the stimulus, was removed to eliminate the stimulus signal. The windowed data was then filtered using $24 \mathrm{~dB}$ per octave Butterworth high and low pass filters with corner frequencies of $1.6 \mathrm{kHz}$ and $2.8 \mathrm{kHz}$, respectively. This filtering preserved the $1.5-3.0 \mathrm{kHz}$ octave band, in which most of the useful OAE information is contained. Lower frequencies, which are often contaminated by noise, and higher frequencies, which are sometimes contaminated by "ringing", were attenuated.

The test was based on almost 512 responses recorded after the stimulus.

The test was made for three times for each ear to value the exam's repeatability, and was performed in a sleeping well-fed neonate; no sedation was required.

When TEOAE $E_{S}$ were not recorded in one or both ears, the babies were considered to fail so that finally were obtained four groups of newborns each one with its secondary steps (Table 1 ):

I. PASS without risk factor: free to go home without advice for the parents.

II. PASS with risk factor: retest at the age of 7 months (ECHOCHECK).

Table 2 Groups of the born

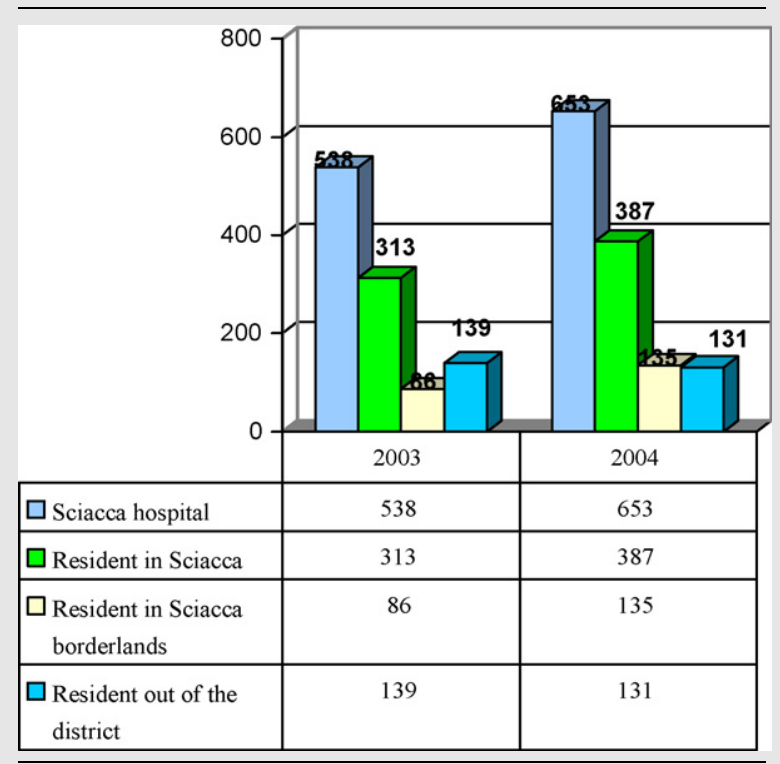


Table 3 Percentage of screened newborns in the hospital

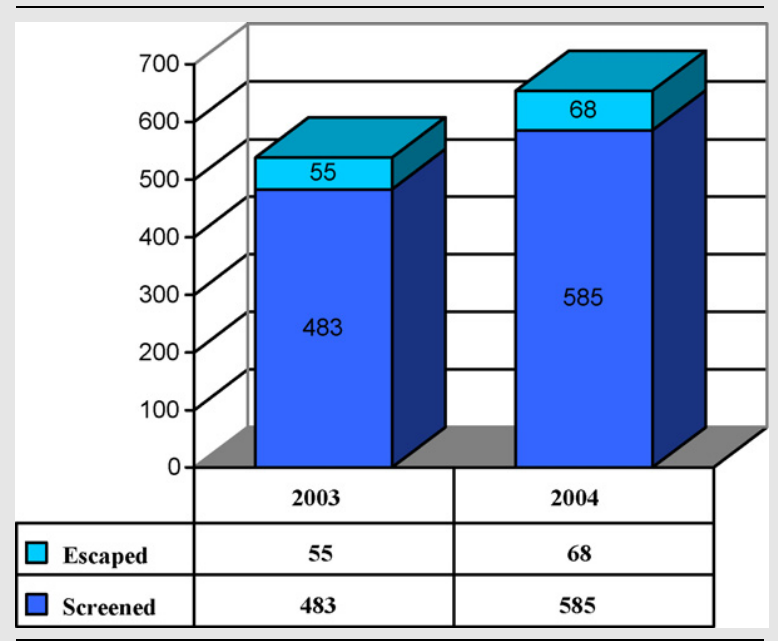

III. FAIL without risk factor: re-screening (ECHO(HECK) after 2 weeks for a maximum of four times and those who failed were referred for follow-up at the age of 3.5 months to the Speech and Hearing Centre.
IV. FAIL with risk factor: retest after 2 weeks (ECHOCHECK) and in case of fail at rescreen the neonate was immediately send to the Speech and Hearing Centre.

The audiological evaluation consisted of: TEOAE and AABR.

\section{Results}

In the years 2003-2004 the number of the newborns in the Sciacca hospital was 1191 and of all 1068 underwent screening; all the infants were divided in three groups:

(1) resident in Sciacca;

(2) resident in Sciacca borderlands;

(3) resident out of the district of Sciacca (Table 2).

In the 2003 the newborns born in the hospital of Sciacca were 538 and the number of infants whose underwent screening was $483(90 \%)$ with a 10\% (55 neonates) of escaped.

Table 4 Distribution of the born

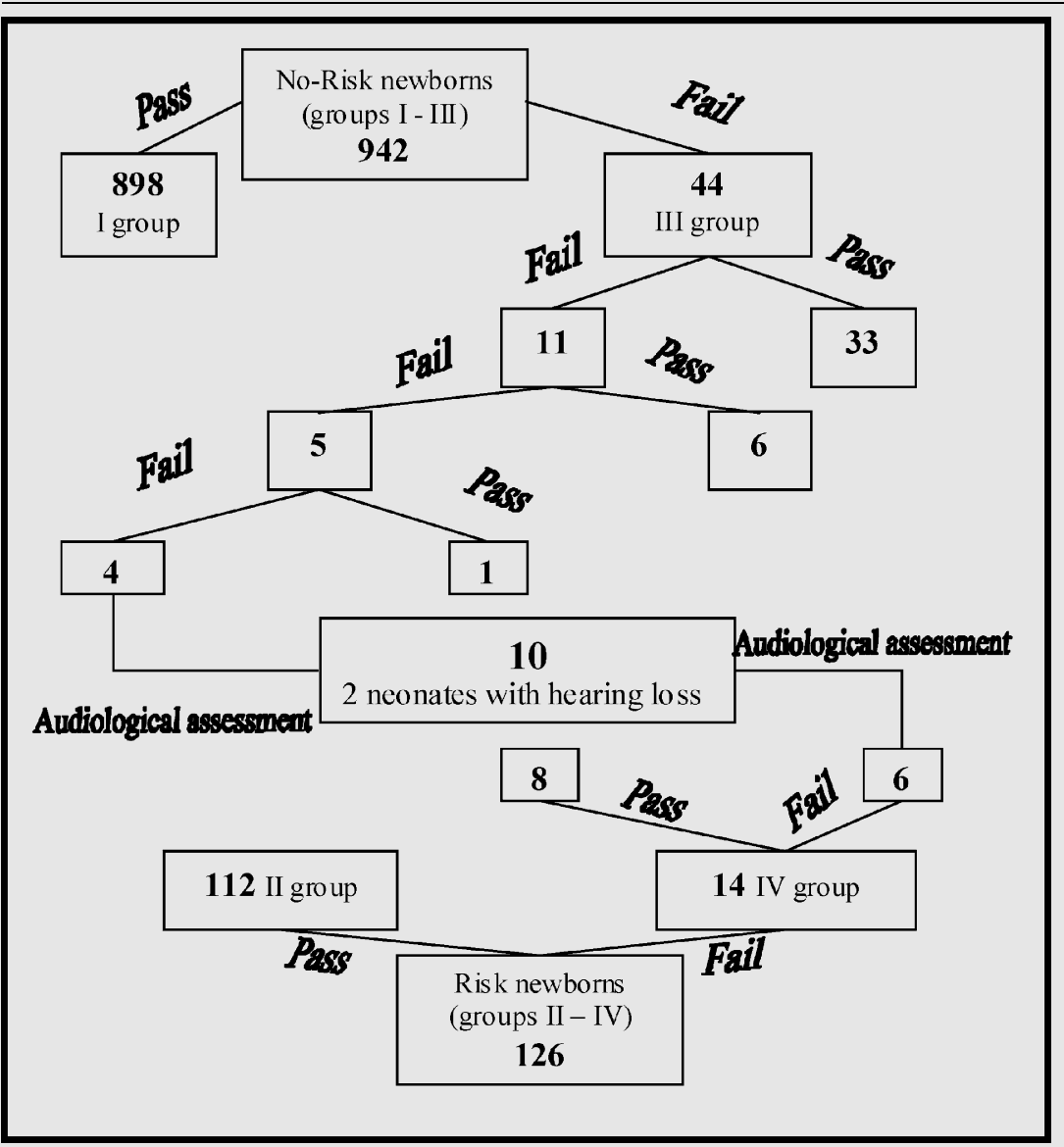


In the 2004 the number of newborns born in the hospital of Sciacca was 653 and $90 \%$ of all (585 infants) underwent screening while 10\% (68 neonates) escaped (Table 3).

The infants with a bilateral sensorineural hearing loss identified were one in 2003 and one in 2004; the incidence of sensorineural hearing loss was estimated to be $2.07 / 1000$ in 2003 and $1.70 / 1000$ in 2004 .

From the total number of neonates screened (1068), 1010 (898 of the I group and 112 of the II group) passed the first screening step while 58 infants failed; from 58 examined in the second screening step 17 failed and 6 of them (IV group) underwent a audiologic assessment while 11 (III group) were examined in a third step. Five newborns failed at the third step. The specified value was $95 \%$ at the first step but became $99 \%$ after the second step.

Ten infants underwent a comprehensive audiologic assessment to confirm the existence of hearing loss. Two of these (one in 2003 and one in 2004) were confirmed to have a congenital hearing loss. All these neonates had a moderately severe (56$70 \mathrm{~dB}$ ) bilateral sensorineural hearing loss and began a rehabilitation program before the age of 5 months (Table 4).

The false positive of this protocol were eight with a $99.6 \%$ final specificity.

\section{Discussion and conclusions}

Universal newborn hearing screening results in earlier diagnosis of hearing loss. The diagnosis of hearing loss within the first few months of life allows the opportunity to begin early intervention services for families with infants.

Yoshinaga-Itano, Coulter and Thomson in a study of 294 children with congenital hearing loss, found that children born in a Colorado hospital which had a newborn hearing screening program were identified earlier than those born in hospitals without UNHS programs. They also began early intervention by two months of age and had an $80 \%$ probability of having language development within the normal range of development for children assessed between 12 and 60 months of age [34].

According to Yoshinaga-Itano, to Peck and to Pujol et al. [22,23,34] we felt the necessity to develop and propose a screening programme, using TOAE methods that have many advantages.

In fact in case of a newborn PASS with risk factor (II group) the possibility of false negative could be overcome advising the parents to take care to infant's speech-hearing develop and repeating a new test at the age of 7 months.
Moreover, to have a good "screen sensitivity" (the proportion of the children tested with a specified condition, detected by the screening protocol) and a good "screen specificity" (the proportion of the children tested who do not have a specified condition who are correctly identified by the screen protocol) we took care, in particular, to the infants belonging to third group (FAlL without risk factor). This was the group with a certain numbers of false positive observed. In fact in presence of a newborn "FAIL without risk factor", the choice of repeat the exam after 15 days for a maximum of four times reduces the number of more expensive secondary level exams, limiting only in rare cases the visit in a speech and hearing centre, but at the same time, it gives to the deafness infant, the possibility to undergo an audiologic assessment at the age of 3.5 months.

Finally the choice to divide all the infants into four groups let us reduce the percentage of false positive that was only $0.74 \%$ (eight newborns).

All the infants with a diagnosis of sensorineural hearing loss began a rehabilitation programme before the age of 5 months and they have a good speech development and speech intelligibility.

\section{References}

[1] B.R. Vohr, K.R. White, A.B. Maxon, M.J. Johnson, Factor affecting the interpretation of transient evoked otoacoustic emission results in neonatal hearing screening, 1993-1996, J. Pediatr. 133 (3) (1998) 353-357.

[2] H.M. Fortnum, A. Davis, A. Butler, J. Stevens, Health service implications of changes in aetiology and referral patterns of hearing-impaired children in Trent 1985-1993, in: Report of the MRC Institute of Hearing Research, IHR, Nottingham, 1997.

[3] D. Nekahm, V. Weichbold, K. Welzl-Müller, Epidemiology of permanent childhood hearing impairment in the Tyrol, 1980-1994, Scand Audiol. 30 (2001) 197-202.

[4] H.M. Fortnum, Epidemiology of permanent childhood hearing impairment, Audiol. Med. 1 (2003) 155-164.

[5] H.M. Fortnum, Q. Summerfield, D. Marshall, A. Davis, J. Bamford, Prevalence of permanent childhood hearing impairment in the United Kingdom and implications for universal neonatal hearing screening: questionnaire based ascertainment study, Br. Med. J. 323 (2001) 536-539.

[6] C.D. Drews, M. Yeargin-Allsopp, C.C. Murphy, P. Decouflé, Hearing impairment among 10-year-old children: metropolitan Atlanta, 1985 through 1987, Am. J. Pub. Health 84 (7) (1994) 1164-1166.

[7] Z. Naeem, V. Newton, Prevalence of sensorineural hearing loss in Asian children, Br. J. Audiol. 30 (5) (1996) 332-339.

[8] G.J. Sutton, S.J. Rowe, Risk factors for childhood sensorineural hearing loss in the Oxford region, $\mathrm{Br}$. J. Audiol. 31 (1) (1997) 39-54.

[9] T. Pitt, The Epidemiology of Childhood Hearing Impairment in South-east Ireland, National Rehabilitation Board, 1996.

[10] K. Van Naarden, P. Decouflé, K. Caldwell, Prevalence and characteristics of children with serious hearing impairment 
in metropolitan Atlanta, 1991-1993, Pediatrics 103 (3) (1999) 570-575.

[11] R. Morton, V. Sharma, J. Nicholson, M. Broderick, J. Poyser, Disability in children from different ethinc populations, Child: Care, Health and Dev 28 (2002) 87-93.

[12] K.P. Steel, C.J. Kros, A genetic approach to understanding auditory function, Nat. Genet. 27 (2001) 143-149.

[13] M.B. Petersen, Non-syndromic autosomal-dominant deafness, Clin. Genet. 62 (2002) 1-13.

[14] M. Bitner-Glindzicz, Hereditary deafness and phenotyping in humans, Br. M. Bull. 63 (2002) 73-94.

[15] A. Parving, Prevalence of congenital hearing impairment and risk factors, in: F. Grandori (Ed.), European Consensus Conference on Neonatal Hearing Screening, Milan, May 15-16, (1998), pp. 18-23.

[16] H.S. Habib, H. Abdelgaffar, Neonatal hearing screening with transient evoked otoacoustic emissions in Western Saudi Arabia, Int. J. Pediatr. Otorhinolaryngol. 69 (2005) 839-842.

[17] H.M. Robinshaw, Early intervention for hearing impairment: differences in the timing of communication and linguistic development, Br. J. Audiol. 29 (1995) 315-334.

[18] C. Yoshinago-Itano, D. Coulter, V. Thomson. The Colorado newborn hearing screening project: effects on speech and language development for children with hearing loss, in: M.K. Philbin, S.N. Graven, A. Robertson (Eds.), The Influence of Auditory Experience on the Fetus, Newborn, and Preterm Infant: Report of the Sound Study Group of the National Resource Center: The Physical and Development Environment of the High Risk Infant, J. Perinatol. 2000; 20(8:2): S132-S137.

[19] M.P. Moeller, Early intervention and language development in children who are deaf and hard of hearing, Pediatrics 106 (2000) E43.

[20] L. Spivak, H. Sokol, Beyond newborn screening: early diagnosis and management of hearing loss in infants, Adv. Neonatal Care 5 (2) (2005) 104-112.

[21] J.J. Eggermont, Defining and determining sensitive periods, Acta Otolaryngol. (Stockh.) Suppl. 429 (1986) 5-9.
[22] J.E. Peck, Development of hearing. Part II. Embryology, J. Am. Acad. Audiol. 5 (1994) 359-365.

[23] R. Pujol, M. Lavigne-Rebillard, A. Uziel, Development of the human cochlea, Acta Otolaryngol. 482 (Suppl.) (1995) 7-17.

[24] W.K. Frankenburg, J. Chen, S.M. Thornton, Common pitfalls in the evaluation of developmental screening tests, J. Pediatr. 113 (1988) 1110-1113.

[25] National Institutes of Health, Early identification of hearing impairment in infants and young children, NIH Consens. Statement 11 (1) (1993) 1-24.

[26] M. Lutman, F. Grandori, Screening for neonatal defects. European consensus statement, Eur. J. Pediatr. 158 (1999) 95-96.

[27] K. Welzl-Müller, Examples of implemented screening programs, in: F. Grandori (Ed.), European Consensus Conference on Neonatal Hearing Screening, Milan, May 15-16, (1998), pp. $53-56$.

[28] M.S. Robinette, Methods of infant screening, in: F. Grandori (Ed.), European Consensus Conference on Neonatal Hearing Screening, Milan, May 15-16, (1998), pp. 24-35.

[29] C. Kennedy, L. Kimm, D. Cafarelli-Dees, M. Campbell, R. Thornton, Early identification of permanent childhood hearing impairment: a controlled trial of universal neonatal screening, Lancet 352 (1998) 1957-1964.

[30] P.M. Watkin, Neonatal screening-methods and outcome, Audiol. Med. 1 (2003) 165-174.

[31] E.M. Mäki-Torkko, P.K. Lindholm, M.R.H. Väyrynen, J.T. Leisti, M.J. Sorri, Epidemiology of moderate to profound childhood hearing impairments in northern Finland. Any changes in ten years? Scand. Audiol. 27 (1998) 95-103.

[32] Joint Committee on Infant Hearing, Year 2000 position statement: principles and guidelines for early hearing detection and intervention programs, Am. J. Audiol. 9 (2000) 9-29.

[33] E.M. Mäki-Torkko, Current issues on aetiological evaluation of hearing-impaired infants, Audiol. Med. 1 (2003) 185-190.

[34] C. Yoshinaga-Itano, Universal newborn hearing screening programs and development outcomes, Audiol. Med. 1 (2003) 199-206.

Available online at www.sciencedirect.com 